\title{
DFT Approach on Characterizing Various Configurations of Carbon Monoxide Uptake by a Single-Standing Iron- Doped Carbon Layer Surface
}

\author{
Kun Harismah ${ }^{1, *}$ (D) , Hasan Zandi ${ }^{2} \mathbb{D}$ \\ 1 Department of Chemical Engineering, Faculty of Engineering, Universitas Muhammadiyah Surakarta, Surakarta, \\ Indonesia; kun.harismah@ums.ac.id (K.H.); \\ 2 Department of Chemistry, Faculty of Science, University of Qom, Qom, Iran; hasan_zandi@yahoo.com (H.Z.); \\ * Correspondence: kun.harismah@ums.ac.id;
}

Scopus Author ID 56982926300

Received: 4.05.2021; Revised: 2.06.2021; Accepted: 3.06.2021; Published: 9.06.2021

\begin{abstract}
Density functional theory (DFT) calculations were carried out to characterize various configurations of carbon monoxide ( $\mathrm{CO}$ ) uptake by a single-standing iron-doped (Fe-doped) carbon (FC) layer surface. Different starting positions of $\mathrm{CO}$ were examined towards Fe of FC layer leading to C-Head, O-Head, and P-Head models of interacting CO@FC complex formations. Optimization processes and properties evaluations all indicated that the FC layer surface could work as a diagnosis sensor in addition to its role of $\mathrm{CO}$ uptake for deathful gas removal purposes. All results indicated that the $\mathrm{C}$-Head model could be the most suitable configuration for $\mathrm{CO}$ uptake by FC layer surface. All other two models were also suitable for the purpose. The evaluated infrared (IR) spectra demonstrated variations of locations of peaks in the models in agreement with changes of bond distances after $\mathrm{CO}$ uptake. Molecular orbitals features also indicated that the FC layer models were suitable for CO uptake, in which such representations of orbitals distribution patterns approved the effects of the formation of interacting CO@FC complexes. Consequently, the dual role of diagnosis sensor and gas removal could be proposed for $\mathrm{FC}$ layer surface during $\mathrm{CO}$ uptake processes to further investigate the important issues of this deathful gas.
\end{abstract}

Keywords: carbon monoxide; carbon layer; gas uptake; surface; DFT.

(C) 2021 by the authors. This article is an open-access article distributed under the terms and conditions of the Creative Commons Attribution (CC BY) license (https://creativecommons.org/licenses/by/4.0/).

\section{Introduction}

The innovation of nanotechnology has raised the attention of researchers on developing applications for related nanostructures in various fields, especially those applicable in health care systems [1-3]. In this regard, several other types of nanostructures have been introduced in addition to the pioneering carbon nanotube (CNT) with differences in shapes and compositions [4-6]. Adsorption applications have been seen as important for nanostructures because of their proper surface interaction with other substances [7-9]. Several attempts have been dedicated to exploring such function for nanostructures in living systems, for example, in drug delivery purposes for carrying medicinal compounds up to correct target tissue [10-12]. Outside living systems, further applications have still been developed for nanostructures in health care systems, such as sensing pollutants and their removal from the environment [1315]. Harmful gases are always available in the environment of current industrial cities with serious negative impacts on human health quality [16]. It is impossible to get rid of such 
harmful gases easily; therefore, investigating possible solutions for harmful gases sensing and removal has always been an important topic of research, especially nanostructures [17-19]. Carbon monoxide $(\mathrm{CO})$ is one of the deathful pollutant gases with several human victims every year in addition to its temporarily poisoning [20]. In addition to widely spreading $\mathrm{CO}$ from industrial exhausts, it is also widely produced in burning hydrocarbons in lack of enough oxygen even for hydrocarbon-based heating systems of homes leading to asleep death by $\mathrm{CO}$ gas ultra-poisoning [21]. Although considerable efforts of researchers have been devoted to innovating a facile way of sensing CO gas and removing the environment, the works did not reach a final solution at this time, and further works are still required [22-24]. Graphene, which is a monolayer type of carbon nanostructure, has been used for such $\mathrm{CO}$ gas uptake by earlier works [25]. Indeed, the researchers put their effort into optimizing graphene surface for CO uptake by modifying metal dopants such as iron (Fe) [26]. They indicated that such an idea of $\mathrm{CO}$ uptake could be better achievable by employing a Fe-doped graphene surface in contrast with a pure surface [27]. The lack of study was indeed various unconsidering configuration of adsorbed $\mathrm{CO}$ at the surface by emphasizing only one direction of $\mathrm{Fe}-\mathrm{C}=\mathrm{O}$ adsorption [27]. As an advantage of computer-based works, details of molecular systems could be very well described employing descriptors at molecular and atomic scales [28-32]. Therefore, this work was aimed to use such an advantage for describing various configurations of $\mathrm{CO}$ gas uptake at the surface of a single standing Fe-doped carbon (FC) layer. To this aim, coronene (Figure 1) monolayer of $s p^{2}$ carbon atoms was chosen for mimicking graphene surface on a molecular scale [33]. In addition to pure nanostructures, earlier works indicated that atomic doping and decorating could provide nanostructures with more proper benefits for specified applications [34-36]. Additional of one Fe atom could supply carbon layer surface with more vacant orbitals for adsorbing electrons of other substances in interaction processes; therefore, an advantage of Fe-doped carbon layer surface could be expected for employing in $\mathrm{CO}$ gas uptake.

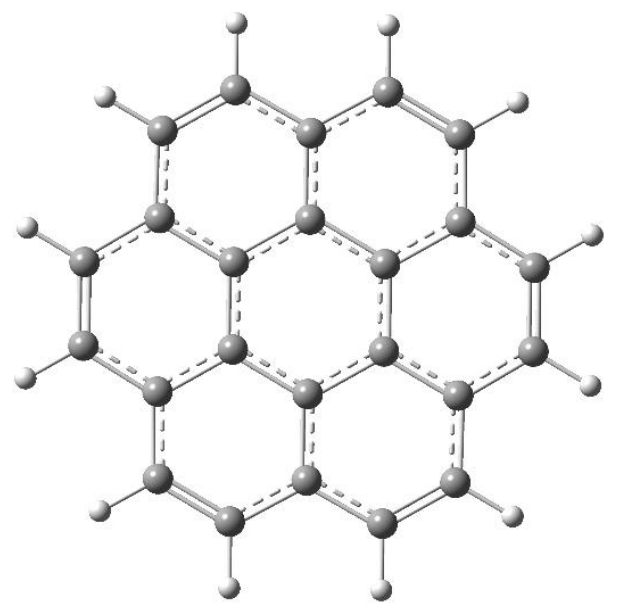

Figure 1. Coronene model.

Within this work, quantum chemical density functional theory (DFT) calculations were performed to obtain optimized structures Fe-doped carbon (FC) layer surface and $\mathrm{CO}$ in singular and complex models (Figure 2) to achieve the purpose of investigating various configurations of $\mathrm{CO}$ at the adsorbent surface. Besides optimized models, quantitative and qualitative descriptors were evaluated to describe the model systems in detail to see what would happen inside such a gas uptake process. All quantitative and qualitative achievements of this work were exhibited in Table 1 and Figures 1-4. 


\section{Materials and Methods}

For providing FC layer surface, coronene model $\left(\mathrm{C}_{24} \mathrm{H}_{12}\right)$, as exhibited in Figure 1, was obtained from ChemSpider [37], and it was doped by one $\mathrm{Fe}$ atom $\left(\mathrm{FeC}_{23} \mathrm{H}_{12}\right)$ to be involved in the $\mathrm{CO}$ gas uptake process. The model was optimized to achieve the minimized energy structure with confirmation by frequency calculations avoiding the existence of any imaginary frequencies. Next, various starting configurations of $\mathrm{CO}$ molecule at the Fe-doped surface were investigated by performing additional optimization processes. Finally, three configurations, including C-head, O-head, and P-head (parallel) models, were obtained for CO uptake by FC layer surface (Figure 2). Each model was analyzed using obtained descriptors consisting of optimized geometries, energy (E), adsorption energy (Eads), energy levels of the highest occupied and the lowest unoccupied molecular orbitals (HOMO and LUMO), energy gap (EG), Fermi energy (FE), dipole moment (DM), and volume (V) as summarized in Table 1. Furthermore, infrared (IR) spectra (Figure 3), HOMO and LUMO distribution patterns, and electrostatic potential (ESP) surfaces (Figure 4) were all visualized for the optimized models. All calculations were performed at the WB97XD/6-31G* level of DFT using the Gaussian program [38].

Table 1. Obtained features for the optimized models.

\begin{tabular}{l|l|l|l|l} 
Feature & FC & C-Head & O-Head & P-Head \\
\hline E eV & -58420.955 & -61504.668 & -61503.882 & -61503.944 \\
\hline Eads eV & n/a & -1.371 & -0.585 & -0.647 \\
\hline Dads & n/a & 1.901 & 2.028 & 1.979 \\
\hline HOMO eV & -6.739 & -6.849 & -6.609 & -6.974 \\
\hline LUMO eV & -0.708 & -0.824 & -0.314 & -0.929 \\
\hline EG eV & 6.031 & 6.025 & 6.295 & 6.045 \\
\hline FE eV & -3.724 & -3.466 & -3.462 & -3.952 \\
\hline DM Debye & 2.264 & 1.553 & 3.527 & 0.664 \\
\hline V cm 3 /mol & 218.267 & 207.678 & 186.975 & 228.133
\end{tabular}

\section{Results and Discussion}

By the importance of harmful gas uptake for diagnosis and removal of hazardous substances from the environment, this work was performed to investigate details of CO uptake by FC layer surface. The models were optimized first to reach the minimum energy levels. Next, details of such systems were discussed by means of evaluated features. As seen by panels of Figure 1, three models were finalized for the relaxation of $\mathrm{CO}$ gas at the $\mathrm{FC}$ layer surface, including C-Head, O-Head, and P-Head. Different starting positions of $\mathrm{CO}$ at the surface were examined to reach these finalized models as the most probable ones of interacting CO@FC complex formations. The visual representations of models could show different geometrical properties, including different bond distances, especially for those bonds close to the interacting region. Atomic dopants could somehow raise features for the doped region, especially for interacting purposes with other substances. Therefore, such a region of the model of this work could play an important role for gas uptake purposes. $\mathrm{CO}$ itself could work as a good ligand with lone pairs of electrons at both of $\mathrm{C}$ and $\mathrm{O}$ atomic sites, in which that of single lone pair of $\mathrm{C}$ atom could be expected to work better than those two lone pairs of $\mathrm{O}$ atom. In this regard, comparing the optimized visual representations could show the formation of almost a chemical covalent bond between $\mathrm{Fe}$ and $\mathrm{CO}$ in $\mathrm{C}$-Head and P-Head models but still a noncovalent interaction for the O-Head model. These results could show the importance of those available vacant orbitals of $\mathrm{Fe}$ and that lone pair of electrons of $\mathrm{C}$ atom of $\mathrm{CO}$ for making 
complexes. Further analyses of bond distances could also show different features for the models with more or less strength for such complexes.

FC>
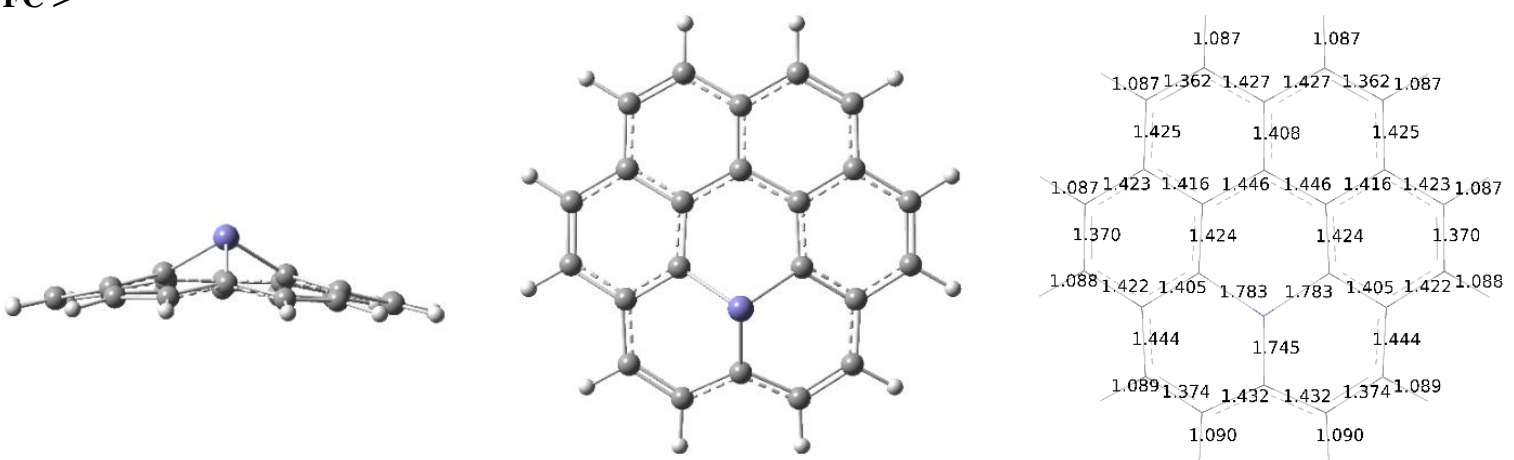

C-Head >
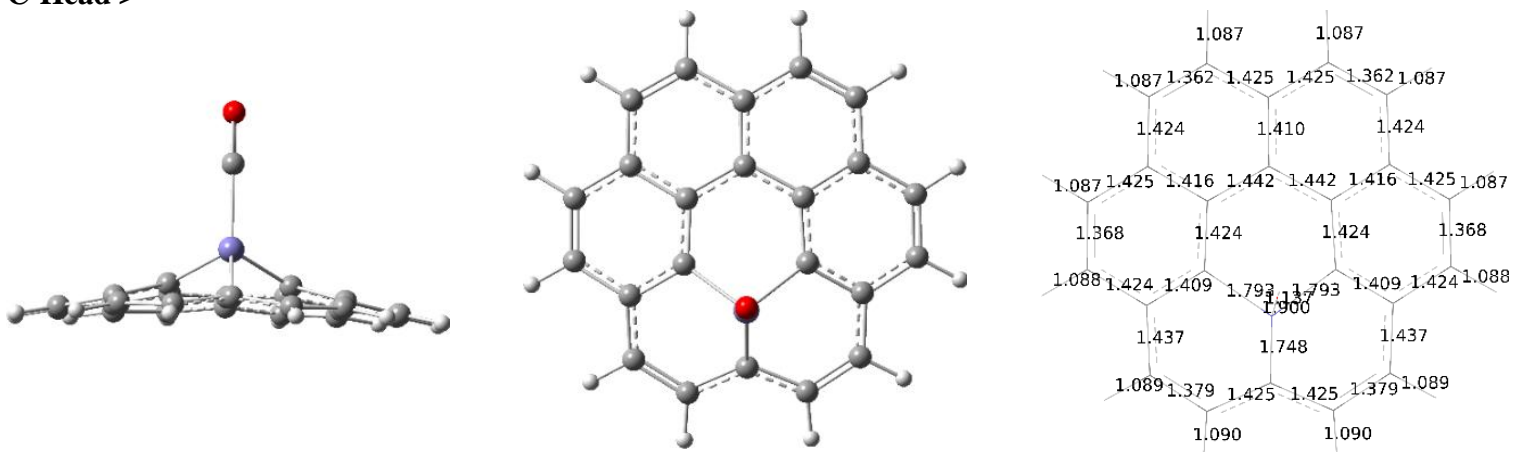

O-Head >
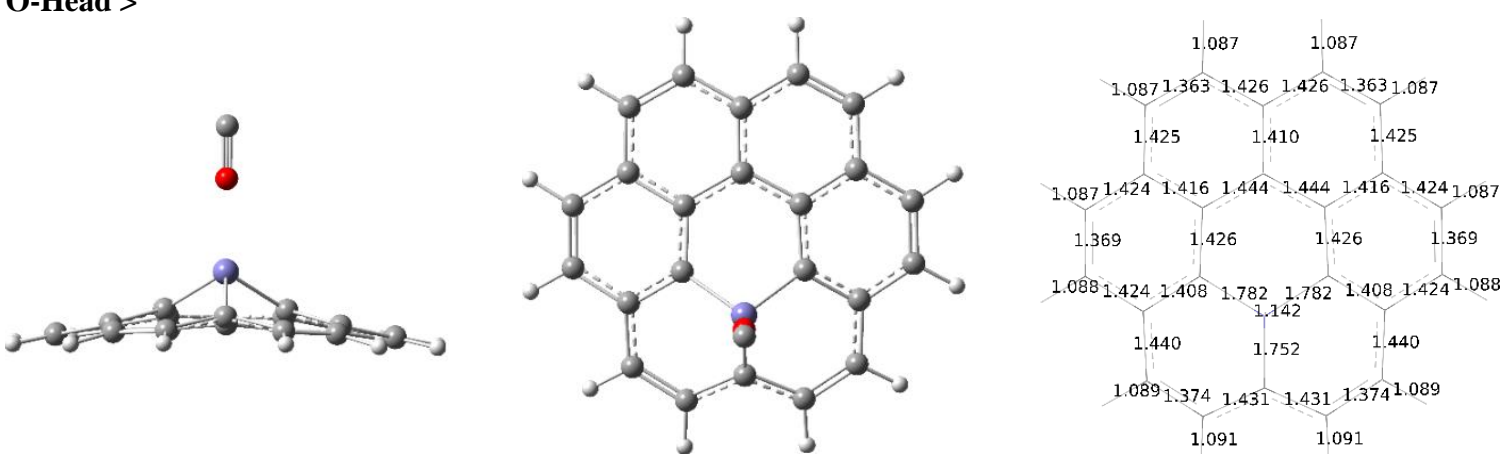

P-Head >
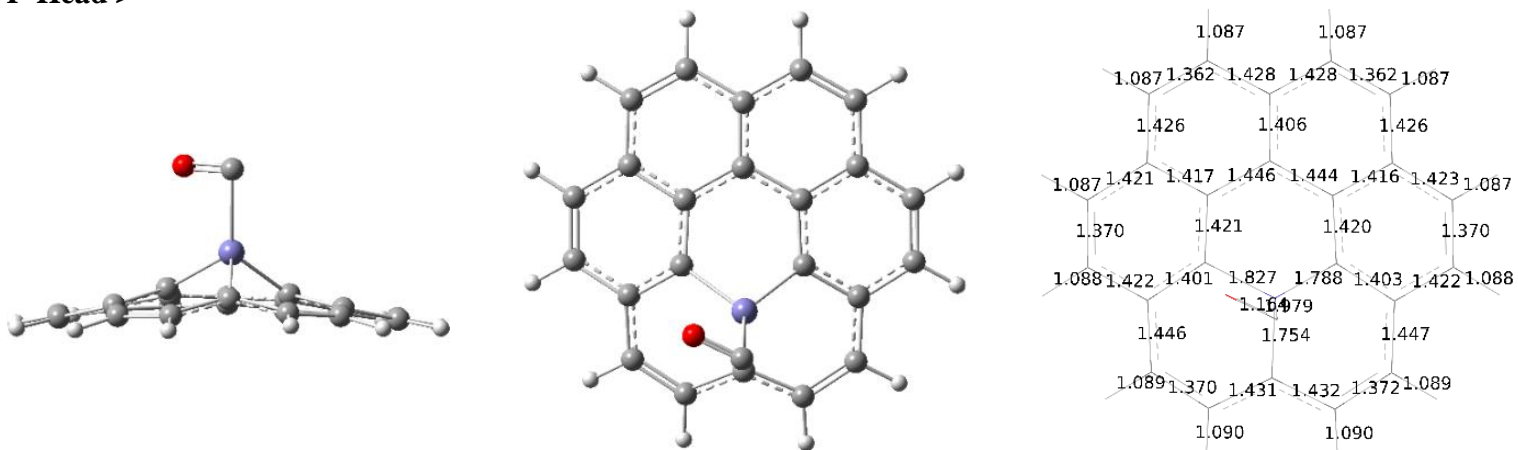

Figure 2. Different views of optimized models of this work including pure Fe-doped carbon (FC) layer, C-Head model with $\mathrm{C}$ of $\mathrm{CO}$ towards Fe of FC layer, O-Head model with $\mathrm{O}$ of $\mathrm{CO}$ towards Fe of FC layer, and P-Head model with parallel $\mathrm{CO}$ with Fe of FC layer.

Results of Table 1 could reveal that the orders of the strength of $\mathrm{CO} @ \mathrm{FC}$ complexes could be defined by obtained values of E as C-Head > P-Head > O-Head models. In this regard, released values of Eads also approved such achievement with higher adsorption energy for $\mathrm{C}$ Head, and lower one for O-Head model. It was indeed very much interesting that variations of molecular relaxations at the surface of the FC layer yielded different geometrical stabilities for 
the same interacting surface and substance but with different interacting geometries. Distances of such adsorbing interactions were also different by the orientation of $\mathrm{CO}$ configuration towards the Fe atoms of the surface with the shortest distance of $1.901 \AA$ for the $\mathrm{C}$-Head model and the longest distance of $2.028 \AA$ for the O-Head model. The obtained IR spectra (Figure 2) for the optimized models could show the variation of peaks from the original FC layer up to each of the CO uptake models by shifting the peaks to different fields in the CO@FC complexes. In this case, careful recognition of IR spectra could also approve different interaction types among the model systems. These achievements could actually highlight the importance of knowledge about details of materials for extending their further developments and applications.
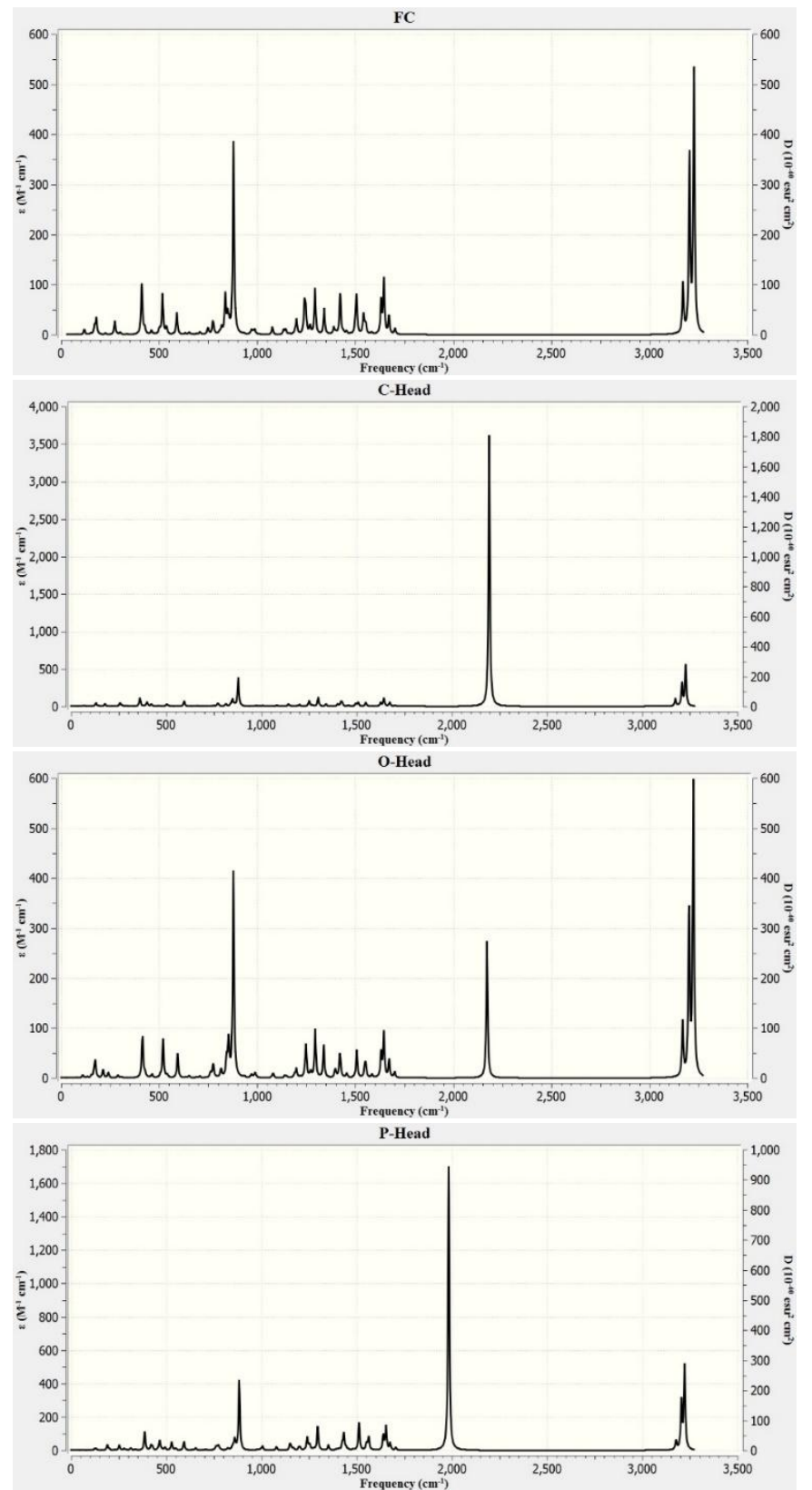

Figure 3. IR spectra for the optimized models.

The obtained values of energy levels for HOMO and LUMO of the models could show the effects of $\mathrm{CO}$ uptake by the FC layer surface, in which measuring values of each of EG and FE could somehow lead to diagnosis sensor for the existence of deathful $\mathrm{CO}$ gas in the environment. Further examinations of orbital features could be held by analyzing 
representations of distribution patterns of HOMO and LUMO and also ESP. The visualized result could show significant effects of $\mathrm{CO}$ uptake on orbital features of the FC layer yielding complexes with new electronic features. Such localization and expansion of orbital features could reveal insightful information about the models' interacting parts and shared electrons between interacting substances. Although HOMO was distributed almost at the whole surface, LUMO was mainly distributed at the Fe-doped region, assigning Fe atoms' important role with vacant orbitals to attract electrons of $\mathrm{CO}$ substances. Hence, molecular orbital features approved that the FC layer could work for $\mathrm{CO}$ uptake by means of available LUMO feature of Fe-doped region; details of such CO uptake showed different characterizations for CO@FC complex formations. To this point, variations of such uptake processes also yielded different values for DM and V for each of C-Head, O-Head, and P-Head models. As a consequence, several factors for the characterization of such $\mathrm{CO}$ uptake process by the FC layer were required.
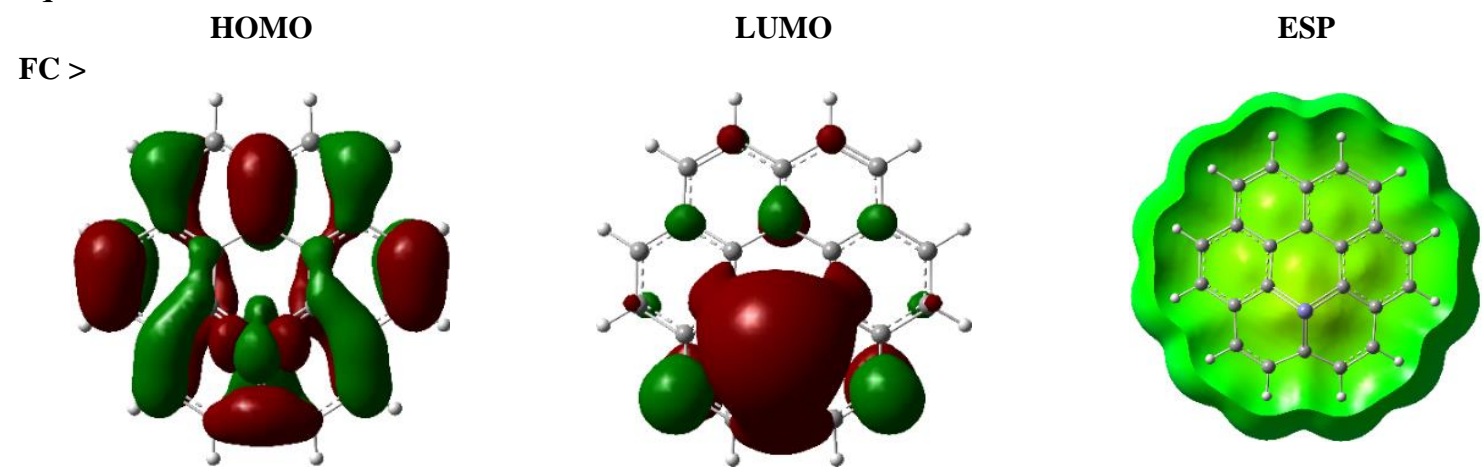

C-Head >
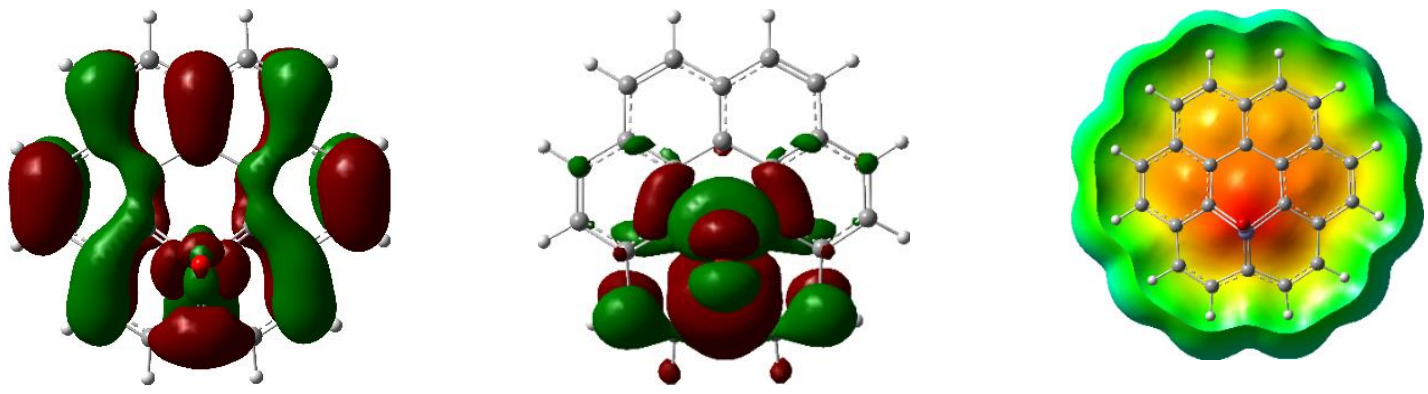

O-Head >
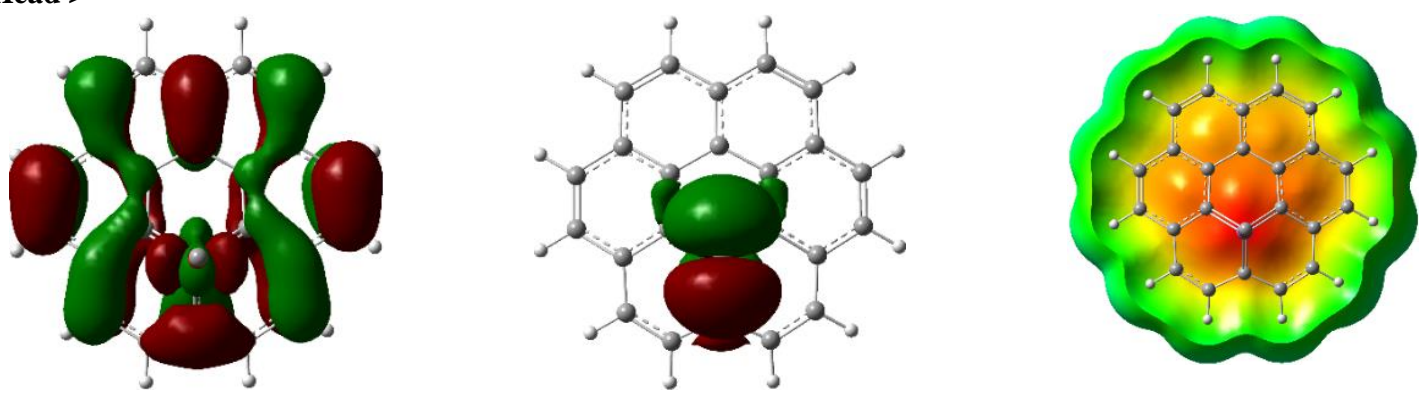

P-Head >
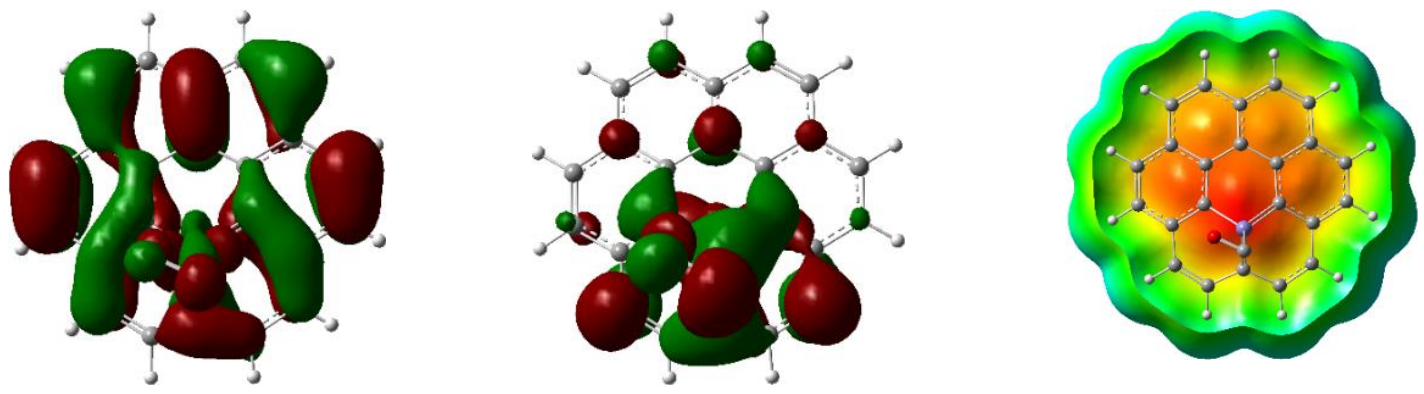

Figure 4. HOMO and LUMO distribution patterns and ESP surfaces for the optimized models. 


\section{Conclusions}

This work was performed to characterize various configurations of $\mathrm{CO}$ uptake by FC layer surface through performing DFT calculations. To approach the purpose of this work, all possible starting positions of $\mathrm{CO}$ towards $\mathrm{Fe}$ of $\mathrm{FC}$ layer were examined, reaching three finalized models of C-Head, O-Head, and P-Head models. The results indicated different stabilities for the models assigning the highest stability for C-Head and the lowest stability for the O-Head model. In both of C-Head and P-Head models, the $\mathrm{C}$ atom was located towards the Fe atom leading to proper interaction with the lone pair of $\mathrm{C}$ of $\mathrm{CO}$ substance and the vacant orbitals of $\mathrm{Fe}$ of the FC layer. Further analyses of interacting energies also approved such achievements for the model systems. In addition to energies, different distances for those bonds especially close to the Fe region were observed for the interacting CO@FC complexes, which were confirmed by the evaluated IR spectra. Details of such mechanisms were clarified by analyzing molecular orbital features by localizing LUMO of the layer, especially at the Fe atom region due to the availability of vacant orbitals. Consequently, such an FC layer surface could work as a diagnosis sensor in addition to the role of $\mathrm{CO}$ uptake for removal processes. Details showed that the FC layer surface could adsorb all configurations of $\mathrm{CO}$ but with different strengths of such interacting CO@FC complex formations.

\section{Funding}

This research received no external funding.

\section{Acknowledgments}

This research has no acknowledgment,

\section{Conflicts of Interest}

The authors declare no conflict of interest.

\section{References}

1. Harismah, K.; Mirzaei, M.; Da'I, M.; Roshandel, Z.; Salarrezaei, E. In silico investigation of nanocarbon biosensors for diagnosis of COVID-19. Eurasian Chemical Communications 2021, 3, 95-102, https://doi.org/10.22034/ecc.2021.267226.1120.

2. Mirzaei, M.; Gulseren, O.; Rafienia, M.; Zare, A. Nanocarbon-assisted biosensor for diagnosis of exhaled biomarkers of lung cancer: DFT approach. Eurasian Chemical Communications 2021, 3, 154-161, https://doi.org/10.22034/ecc.2021.269256.1126.

3. Mirzaei, M. Nanotechnology for science and engineering. Advanced Journal of Science and Engineering 2020, 1, 67-68, https://doi.org/10.22034/AJSE2013067.

4. Iijima, S. Carbon nanotubes: past, present, and future. Physica $B$ 2020, 323, 1-5, https://doi.org/10.1016/S0921-4526(02)00869-4.

5. Alsinai, A.; Alwardi, A.; Farahani, M.R.; Soner, N.D. On the $\psi$ k-polynomial of graph. Eurasian Chemical Communications 2021, 3, 219-226, http://doi.org/10.22034/ecc.2021.274069.1138.

6. Shamsin Beyranvand, H.; Sarlak, H. Effects of graphene oxide (GO) nanoparticle and SDS on storage capacity of $\mathrm{CO} 2$ in hydrate. Eurasian Chemical Communications 2021, 3, 233-243, https://doi.org/10.22034/ecc.2021.270619.1129.

7. Tahmasebi, E.; Shakerzadeh, E. Potential application of B40 fullerene as an innovative anode material for Ca-ion batteries: in silico investigation. Lab-in-Silico 2020, 1, 16-20.

8. Ariaei, S. DFT approach on arsine and phosphine gases adsorption at the surface of B16C16 nanocluster. Lab-in-Silico 2020, 1, 44-49. 
9. Fallahpour, F.; Ariaei, S. Computational investigation of B6 particle for H2S capturing. Advanced Journal of Science and Engineering 2021, 2, 31-35, https://doi.org/10.22034/advjscieng21021031.

10. Afshar, M.; Ranjineh Khojasteh, R.; Ahmadi, R. Adsorption of lomustin anticancer drug on the surface of carbon nanotube: a theoretical study. Eurasian Chemical Communications 2020, 2, 595-603, https://doi.org/10.33945/SAMI/ECC.2020.5.5.

11. Sherafati, M.; Rad, A.S.; Ardjmand, M.; Heydarinasab, A.; Peyravi, M.; Mirzaei, M. Beryllium oxide (BeO) nanotube provides excellent surface towards adenine adsorption: a dispersion-corrected DFT study in gas and water phases. Current Applied Physics 2018, 18, 1059-1065, https://doi.org/10.1016/j.cap.2018.05.024.

12. Mirzaei, M.; Yousefi, M. Computational studies of the purine-functionalized graphene sheets. Superlattices and Microstructures 2012, 52, 612-617, https://doi.org/10.1016/j.spmi.2012.06.027.

13. Ren, X.; Qu, R.; Liu, S.; Zhao, H.; Wu, W.; Song, H.; Zheng, C.; Wu, X.; Gao, X. Synthesis of zeolites from coal fly ash for the removal of harmful gaseous pollutants: a review. Aerosol and Air Quality Research 2020, 20, 1127-1144, https://doi.org/10.4209/aaqr.2019.12.0651.

14. Zhou, J.; Wang, H. Study on efficient removal of SOx and NOx from marine exhaust gas by wet scrubbing method using urea peroxide solution. Chemical Engineering Journal 2020, 390, 124567, https://doi.org/10.1016/j.cej.2020.124567.

15. Jalali Sarvestani, M.R.; Ahmadi, R. Adsorption of TNT on the surface of pristine and N-doped carbon nanocone: a theoretical study. Asian Journal of Nanosciences and Materials 2020, 3, 103-114, https://doi.org/10.26655/AJNANOMAT.2020.2.2.

16. Battaglia, M.; Thomason, W.; Fike, J.H.; Evanylo, G.K.; von Cossel, M.; Babur, E.; Iqbal, Y. Diatta, A.A. The broad impacts of corn stover and wheat straw removal for biofuel production on crop productivity, soil health and greenhouse gas emissions: a review. GCB Bioenergy 2021, 13, 45-57, https://doi.org/10.1111/gcbb.12774.

17. Xiong, F.; Rother, G.; Gong, Y.; Moortgat, J. Reexamining supercritical gas adsorption theories in nanoporous shales under geological conditions. Fuel 2021, 287, 119454, https://doi.org/10.1016/j.fuel.2020.119454.

18. Wu, Y.; Huang, N.; Wang, J. Sensitive characteristics of ZnO nano gas sensor based on dynamic temperature modulation. Results in Physics 2020, 18, 103241, https://doi.org/10.1016/j.rinp.2020.103241.

19. Zahedi, H.; Yousefi, M.; Mirzaei, M. DFT investigation of AlP-doped BN nanotube for CO gas capturing. Lab-in-Silico 2020, 1, 38-43.

20. Domingo, J.L.; Rovira, J. Effects of air pollutants on the transmission and severity of respiratory viral infections. Environmental Research 2020, 109650, https://doi.org/10.1016/j.envres.2020.109650.

21. Mattiuzzi, C.; Lippi, G. Worldwide epidemiology of carbon monoxide poisoning. Human \& Experimental Toxicology 2020, 39, 387-392, https://doi.org/10.1177/0960327119891214.

22. Sam Jebakumar, J.; Juliet, A.V.; Palladium-doped tin oxide nanosensor for the detection of the air pollutant carbon monoxide gas. Sensors 2020, 20, 5889, https://doi.org/10.3390/s20205889.

23. Yang, Q.; Shen, H.; Liang, Z. Analysis of particulate matter and carbon monoxide emission rates from vehicles in a Shanghai tunnel. Sustainable Cities and Society 2020, 56, 102104, https://doi.org/10.1016/j.scs.2020.102104.

24. Sheikhsoleimani, N.; Rouhani, M.; Saeidian, H. Computational evaluation of Ga-doped phagraphene as a work function-based sensor for detection of some air pollutant molecules. Inorganic Chemistry Communications 2020, 118, 107997, https://doi.org/10.1016/j.inoche.2020.107997.

25. Tiwari, S.K.; Sahoo, S.; Wang, N.; Huczko, A. Graphene research and their outputs: Status and prospect. Journal of Science: Advanced Materials and Devices 2020, 5, 10-29, https://doi.org/10.1016/j.jsamd.2020.01.006.

26. Li, D.; Li, W.; Zhang, J. Fe doped BN monolayer: A promising low-cost single atom catalyst for promoted CO oxidation activity. Applied Surface Science 2020, 525, 146567 , https://doi.org/10.1016/j.apsusc.2020.146567.

27. Xu, X.Y.; Xu, H.; Guo, H.; Zhao, C. Mechanism investigations on CO oxidation catalyzed by Fe-doped graphene: a theoretical study. Applied Surface Science 2020, 523, 146496, https://doi.org/10.1016/j.apsusc.2020.146496.

28. Mirzaei, M. Making sense the ideas in silico. Lab-in-Silico 2020, 1, 31-32.

29. Hajhoseinzadeh, K.; Ghiasi, R.; Marjani, A. A computational investigation on the stability and properties of the various isomers of [B7]-anion. Eurasian Chemical Communications 2020, 2, 78-86, https://doi.org/10.33945/SAMI/ECC.2020.1.8. 
30. Gunaydin, S.; Ozkendir, O.M. XAFS calculations of Nd-substituted LiFeO2 material. Lab-in-Silico 2020, 1, 56-60.

31. Novin, N.; Shameli, A.; Balali, E. Molecular dynamics simulation of natural gas sweetening by monoethanolamine. Eurasian Chemical Communications 2020, 2, 619-625, https://doi.org/10.33945/SAMI/ECC.2020.5.8.

32. Shahzad, H.; Ahmadi, R.; Najafpour, J.; Adhami, F. Adsorption of cytarabine on the surface of fullerene C20: a comprehensive DFT study. Eurasian Chemical Communications 2020, 2, 162-169, http://doi.org/10.33945/SAMI/ECC.2020.2.1.

33. Júnior, M.L.P.; Neto, B.G.E.; Giozza, W.F.; Júnior, R.T.S.; e Silva, G.M.; Júnior, L.A.R. Transport of quasiparticles in coronene-based graphene nanoribbons. Journal of Materials Chemistry C 2020, 8, 1210012107, https://doi.org/10.1039/D0TC01319F.

34. Ozkendir O.M. Electronic structure study of Sn-substituted InP semiconductor. Advanced Journal of Science and Engineering 2020, 1, 7-11.

35. Cui, H.; Yan, C.; Jia, P.; Cao, W. Adsorption and sensing behaviors of SF6 decomposed species on Ni-doped C3N monolayer: A first-principles study. Applied Surface Science 2020, 512, 145759, https://doi.org/10.1016/j.apsusc.2020.145759.

36. Hu, J.; Zhao, L.; Du, J.; Jiang, G. Adsorption of rare gases on pristine and doped phosphorene. Applied Surface Science 2020, 504, 144326, https://doi.org/10.1016/j.apsusc.2019.144326.

37. Pence, H.E.; Williams, A. ChemSpider: an online chemical information resource. Journal of Chemical Education 2010, 87, 1123-1124, https://doi.org/10.1021/ed100697w.

38. Frisch, M.; Trucks, G.; Schlegel, H.; Scuseria, G.; Robb, M.; Cheeseman, J.; Montgomery Jr, J.; Vreven, T.; Kudin, K.; Burant, J. Gaussian 09 D.01 Program. Gaussian. Inc.: Wallingford, CT, USA. 2009. 